\title{
Análise de dor e funcionalidade de pacientes com distúrbios osteomioarticulares de membros inferiores
}

\author{
Analysis of pain and functionality \\ of patients with osteomioarticular \\ disorders of lower limbs
}

FisiSenectus . Unochapecó Ano 7, n. 1- Jan/Jun. 2019 p. $49-60$

Cristiane Luci Weber. cristianeluciweber@gmail.com

Fisioterapeuta. Mestre em Gerontologia Biomédica pela Pontifícia Universidade Católica do RS (PUCRS). Professora na área de Fisioterapia Músculo-esquelética e Envelhecimento Humano e orientadora de estágio curricular na Universidade de Caxias do Sul (UCS/RS).

Paula Parenti. paula_parenti@yahoo.com.br

Graduanda do Curso de Fisioterapia da Universidade de Caxias do Sul (UCS/RS).

\section{Resumo}

Introdução: os distúrbios do sistema muscular acarretam dor e alteração da funcionalidade. A qualidade de vida é prejudicada e a execução das atividades de vida diária torna-se restrita. Objetivos: caracterizar os pacientes com distúrbios osteomioarticulares de membros inferiores, incluindo as condições de funcionalidade e intensidade da dor ao início da intervenção fisioterapêutica e analisar fatores sociodemográficos e aspectos inerentes aos atendimentos prestados. Metodologia: estudo do tipo retrospectivo, por meio da análise descritiva de prontuários dos pacientes em processo de reabilitação no setor de traumatologia, pelo serviço de fisioterapia no Centro Clínico da Universidade de Caxias do Sul/Reabilitação (CECLIN-UCS/REAB). Os dados foram coletados nos meses outubro e novembro de 2018. A amostra foi composta de 44 prontuários catalogados, e somente 21 adequaram-se aos critérios de seleção. Para análise de dor e funcionalidade foram examinadas a Escala Visual Analógica (EVA) e a Lower Extremity Functional Scale (LEFS). Resultados: 0 perfil dos pacientes constitui-se por maior predominância do sexo masculino, idade média de $40,14( \pm 16,9)$ anos, número de sessões fisioterapêuticas com média de $17,71( \pm 8,6)$, e média de $5,62( \pm 3,0)$ fisioterapeutas/ estagiários e envolvidos em cada caso. 0 diagnóstico clínico mais prevalente foi de fratura de tíbia e fíbula. A média encontrada na LEFS é de $58,90( \pm 13,8)$ pontos. Conclusão: verificou-se que não há correlação entre a dor e funcionalidade pelas escalas EVA e LEFS $(p=0,548)$. Sugere-se a necessidade de prosseguir o estudo de avaliação dos prontuários, a partir dos dados já existentes e maior número amostral.

\section{Palavras-chave}

Fisioterapia; Tratamento; Reabilitação.

\section{Fisi'enectus}




\begin{abstract}
Introduction: The disorders of the muscular system lead to pain and altered functionality. The quality of life is affected and the performance of the daily activities become limited. Objective: To characterize patients with osteomioarticular disorders of lower limbs, including the functionality conditions and pain intensity at the beginning of the physiotherapeutic intervention, as well as to analyze sociodemographic factors and aspects related to the services provided. Methodology: Retrospective study, carried out through the descriptive analysis of medical records from patients in rehabilitation process in the traumatology sector, by the physiotherapy service at CECLIN-UCS/REAB. The data was collected in October and November 2018. The sample consisted of 44 cataloged records, and only 21 were adapted to the selection criteria. The Visual Analogue Scale (EVA) and Lower Extremity Functional Scale (LEFS) were examined for pain and functional analysis. Results: Patients were more predominantly male, mean age was $40.14( \pm 16,9)$ years, the average number of physiotherapeutic sessions was $17.71( \pm 8,6)$, and mean $5.62( \pm 3,0)$ physiotherapists / trainees and involved in each case. The most prevalent clinical diagnosis was tibia and fibular fracture. The average found in the LEFS was $58.90( \pm 13,8)$ points. Conclusion: It was verified that there is no correlation between pain and functionality by the EVA and LEFS scales $(p=0,548)$. It is suggested that the study of the evaluation of medical records should be continued, based on the existing data and a higher sample size.
\end{abstract}

\title{
Keywords
}

Physiotherapy; Treatment; Rehabilitation.

\section{Introdução}

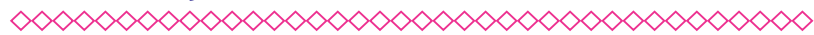

Os distúrbios do sistema muscular que acarretam dor, alteração da funcionalidade e deformidades, estão em segundo lugar quanto à frequência de acometimentos da população em geral que busca atendimento médico. Esses são constituintes de condições incapacitantes e de altos custos para a economia. Tais desordens, em sua totalidade, provocam variados graus de incapacidade funcional e de dor no indivíduo acometido. A qualidade de vida é prejudicada e a execução das atividades de vida diária torna-se restrita. E, muitas vezes, é nessa situação que o indivíduo chega à reabilitação. ${ }^{1-3}$

As mudanças no estilo de vida da população também têm contribuído para a sobrecarga no sistema musculoesquelético, seja por posturas viciosas ou atividades repetitivas no trabalho, ocasionando o comprometimento do sistema muscular, articular e ósseo. Outro ponto a ser analisado são as modificações no padrão demográfico brasileiro, no qual há aumento da expectativa de vida e maior número de idosos, em consequência da redução do crescimento populacional. Dessa forma, é importante reconhecer o perfil epidemiológico do paciente que está em processo de reabilitação no serviço de fisioterapia, para melhor gestão, adequação das práticas de saúde e planejamento das técnicas utilizadas na fisioterapia. ${ }^{4,5,6}$

Visto que a incidência de comprometimentos musculoesqueléticos é alta, torna-se relevante aprofundar os estudos envolvendo o nível de incapacidade que atinge os indivíduos. Dessa forma, o fisioterapeuta é fundamental no campo de atuação da prevenção e reabilitação, para melhora da funcionalidade, analgesia, além de intensificar a recuperação física. ${ }^{1-2}$

Para tais desordens que alteram a funcionalidade e qualidade de vida do paciente, a avaliação é crucial para planejar técnicas adequadas no tratamento fisioterapêutico. Ao avaliar um indivíduo, deve-se englobar a estrutura, função, atividade e participação social, em conformidade com as considerações da Classificação Internacional de Funcionalidade. Questionários autoaplicáveis podem ser incorporados no exame físico durante a avaliação, por possuírem credibilidade, eficiência e baixo custo. ${ }^{7}$

Portanto, este estudo tem como objetivos caracterizar os pacientes com distúrbios osteo- 
mioarticulares de membros inferiores, atendidos por um serviço de fisioterapia em Instituição de Ensino Superior (IES), incluindo as condições de funcionalidade e intensidade da dor ao início da intervenção fisioterapêutica e analisar fatores sócio demográficos e aspectos inerentes aos atendimentos prestados. Pretende ainda subsidiar 0 planejamento de medidas que qualifiquem a intervenção ou contribuir para a solidificação das técnicas e rotinas adotadas na reabilitação, com relação ao quadro álgico e funcionalidade dos pacientes com essas características.

\section{Metodologia}

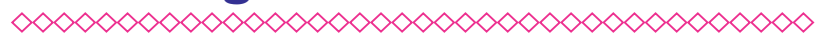

Trata-se de um estudo retrospectivo, realizado por meio da análise descritiva de prontuários dos pacientes em processo de reabilitação (em atendimento ou atendidos desde o início do ano de 2018) no setor de traumatologia, pelo serviço de fisioterapia no Centro Clínico da Universidade de Caxias do Sul/Reabilitação (CECLIN-UCS/REAB), da cidade de Caxias do Sul/RS. A amostra foi composta de 44 prontuários catalogados, apenas 21 adequaram-se aos critérios de seleção da amostra. Não foi realizado cálculo amostral uma vez que se utilizou a totalidade dos prontuários que cumpriram os critérios de inclusão.

Os critérios de inclusão abrangeram prontuários de pacientes que estiveram em processo de reabilitação na referida clínica escola para tratamento de distúrbios osteomioarticulares de membros inferiores, de ambos os sexos, necessariamente atendidos por estudantes matriculados no estágio curricular do Curso de Fisioterapia da Universidade de Caxias do Sul (UCS).

Os dois instrumentos de avaliação de funcionalidade e dor aplicados por ocasião da avaliação, foram: a Escala Visual Analógica (EVA) e o Lower Extremity Functional Scale (LEFS).

Prontuários de pacientes atendidos por distúrbios osteomioarticulares em outros segmentos corporais, ou acolhidos por outros profissionais ou acadêmicos, sem a participação dos estagiários curriculares do Curso de Fisioterapia da UCS e, ainda, prontuários sem registro de um ou ambos os instrumentos citados, foram os critérios de exclusão adotados.

A coleta de dados foi realizada por dois pesquisadores, nos meses de outubro e novembro de 2018, em horário de expediente do serviço. Os dados foram obtidos de uma ficha para a análise dos prontuários elaborada pelos pesquisadores, com informações colhidas a partir da ficha original de avaliação dos pacientes atendidos pelo serviço, incluindo dados de identificação (sexo, idade, diagnóstico clínico, frequência semanal e tempo de atendimento, número de sessões realizadas, número de profissionais e estudantes envolvidos no atendimento). Juntamente foram examinados os parâmetros de avaliação já utilizados na rotina do serviço, anexados no prontuário dos pacientes, incluindo a EVA, utilizada para avaliar a intensidade da dor, e o LEFS, instrumento utilizado para avaliar o estado funcional dos pacientes. ${ }^{8-9}$

A EVA é composta de uma linha horizontal de $10 \mathrm{~cm}$, e engloba desde a extremidade menor apontando para "ausência de dor" até a maior que indica "a pior dor possível", portanto, quando maior a pontuação na EVA, maior será o nível de dor. Utilizando os conceitos do modelo de funcionalidade e incapacidade da Organização Mundial de Saúde (OMS) foi desenvolvida a escala LEFS, que pode ser aplicada a diversos tipos de alterações ortopédicas de membros inferiores. Com base em atividades de vida diária, esse questionário pode ser autoaplicado, com média de 2 minutos para finalizá-lo. Esse instrumento apresenta 20 questões relacionadas com as atividades de vida diária, cada questão pode ser classificada de 0 a 4, partindo de extremamente difícil até nenhuma dificuldade para as atividades, ou seja, quanto maior a pontuação, maior é a funcionalidade do indivíduo. A pontuação varia de 0 a 80 pontos, sendo capacidade funcional máxima, 80 pontos. ${ }^{8-10}$

Para a descrição dos dados, foi utilizada estatística descritiva com distribuição de frequência e percentagem, bem como as medidas de tendência central (média/mediana). A correlação entre as características amostrais e os desfechos avaliados foi realizada por meio da correlação de Spearman. Os softwares utilizados para o teste foram o Excel $^{\circledR}$ e o Programa Estatístico IBM ${ }^{\circledR}$ SPSS Statistics V21 (Statistical Package for the Social Sciences/ 
versão 21). As diferenças foram consideradas significativas quando $p<0,05$.

Este estudo insere-se no projeto de pesquisa intitulado "Atuação fisioterapêutica frente aos distúrbios osteomioarticulares em ambiente ambulatorial", tendo sido submetido e aprovado pelo Comitê de Ética em Pesquisa (CEP) pela Plataforma Brasil, sob o parecer consubstanciado CAAE: 92225418.9.0000.5341. Para a coleta de dados foi respeitado o anonimato dos pacientes, preservando suas identidades, conforme as disposições legais da Resolução n. 466 do ano de 2012, do Conselho Nacional de Saúde.

\section{Resultados}

$\infty<\infty<\infty<\infty<\infty<\infty<\infty<\infty<\infty<\infty<\infty<\infty<\infty<\infty<\infty<\infty<\infty$

Dentre os 44 prontuários catalogados, 21 adequaram-se aos critérios de seleção da amostra, já aqueles com distúrbios osteomioarticulares de outros segmentos corporais, sem prontuários e registro dos instrumentos de avaliação, constituíram os 23 prontuários excluídos da amostra. Dos pacientes analisados, 12 eram do sexo masculino $(57,1 \%)$ e nove do sexo feminino $(42,9 \%)$, (Tabela 1). A faixa etária variou dos 13 a 71 anos, com média de $40,14( \pm 16.9)$ anos. Todos os pacientes foram atendidos em sessões com duração de 40 minutos, e o número semanal de sessões variou de no mínimo dois e no máximo quatro, com média de $2,24( \pm 0,5)$ sessões. Quanto ao número de sessões realizadas, constatou-se uma média de $17,71( \pm 8,6)$ sessões, com o mínimo de três e máximo de 37 sessões por paciente. 0 número de pessoas envolvidas no tratamento de cada um dos pacientes dos prontuários analisados, incluindo profissionais fisioterapeutas, professores do curso de fisioterapia, bolsistas e estagiários curriculares de fisioterapia variou de duas a 14 pessoas, com média de 5,62 $( \pm 3,0)$ pessoas (Tabela 2 ).

Dentre o diagnóstico clínico identificado nos prontuários analisados, o mais frequente, 38,1\%, constituiu-se de fratura de tíbia e fíbula $(n=8)$, seguido por artroplastia de joelho, numa frequência de $23,8 \%(n=5)$. Além desses, citam-se artroplastia de quadril $(n=1)$, fratura de patela $(n=1)$, fratura do pé $(n=1)$, fratura do platô tibial $(n=1)$, instabilidade femoropatelar ( $n=1)$, pós-operatório (PO) de ligamento cruzado anterior e menisco $(n=1)$, PO de reconstrução ligamento femoropatelar $(n=1)$ e PO tenoplastia patelar $(n=1)$. As fraturas de tíbia e fíbula foram mais frequentes nos pacientes do sexo masculino $(n=6)$ e o PO de cirurgias de artroplastia de joelho $(n=3)$, foram mais frequentes no sexo feminino (Tabela 3 ).

Com relação às escalas aplicadas aos pacientes no início do tratamento, anexadas aos prontuários, as pontuações atribuídas pelos pacientes a partir do uso da EVA variaram desde a condição de ausência de dor, até dor insuportável (0 a 10 pontos). Os resultados mediante análise da escala LEFS apresentaram uma média de $58,90( \pm 13,8)$ pontos, com o mínimo de 34 e máximo de 78 pontos dos 80 possíveis.

A Figura 1 descreve a correlação entre a dor e funcionalidade pelas escalas EVA e LEFS, no entanto, não houve resultados significativos $(p=0,548)$.

Ao correlacionar as escalas EVA e LEFS entre os diagnósticos clínicos mais frequentes, sendo esses as fraturas de tíbia e fíbula $(n=8)$ e a artroplastia de joelho $(n=5)$, também não foram encontrados resultados significativos $(p=0,545)$.

Entre os 21 prontuários analisados, cinco pacientes receberam alta, porém somente três prontuários continham as escalas aplicadas ao final do tratamento, e três pacientes abandonaram o tratamento. Considerou-se, portanto, que essa amostra foi insuficiente para realização de análise estatística comparativa.

\section{Discussão}

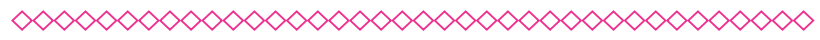

Ao analisar o perfil epidemiológico dos pacientes atendidos no CECLIN-UCS/REAB, verificou-se a predominância do sexo masculino em relação ao feminino, em concordância ao estudo de Silva et al. ${ }^{4}$ que, depois de analisar 274 prontuários na área ortopédica e traumatológica, identificou que $55,5 \%(n=147)$ eram do sexo masculino e $44,5 \%$ $(n=118)$ do sexo feminino. Entretanto, nos resultados dos estudos de Sacon et al. ${ }^{11}$ e Ghisleni et. al. ${ }^{12}$, o sexo feminino teve maior incidência so- 
bre o masculino. Para esses autores, o sexo masculino procura menos os serviços de saúde em relação às mulheres, devido à visão de que o cuidado não é uma prática masculina. Porém tais considerações não se aplicam ao presente estudo. Embora as variáveis profissão/ocupação não tenham sido foco de análise, pode-se sugerir, com base nos resultados encontrados em termos de idade e tipo de lesão, que a ocorrência de fraturas nos membros inferiores esteja relacionada ao predomínio de pacientes do sexo masculino e sua maior exposição aos acidentes laborais e de trânsito.

A média de idade deste estudo foi de 40,14 $( \pm 16,9)$ anos. Portanto, os distúrbios encontrados acometeram indivíduos em idade ativa, mantendo similaridade com a média de idade do estudo de Sacon et al. ${ }^{11}$,que foi de 49,42 $( \pm 15,46)$ anos, e também do autor Cerdeira et al. ${ }^{3}$ que apresentou uma média de idade de 43,7 anos. Visto que a faixa etária dos pacientes acometidos por traumas ortopédicos é altamente produtiva economicamente, essa condição pode ser considerada um problema de saúde pública. Dessa forma, está associada a elevados gastos públicos para a recuperação do indivíduo e custos para a Previdência Social, caso haja incapacidade temporária ou permanente do paciente. ${ }^{13}$

Nota-se que a análise de fatores como o comportamento da dor e funcionalidade frente aos distúrbios osteomioarticulares e a consequente qualificação da intervenção fisioterapêutica, no intuito de agilizar o processo de recuperação do paciente, oportunizam primeiro, a busca da excelência de atendimento, incluindo o controle do quadro álgico e reestabelecimento da funcionalidade do indivíduo; segundo, seu retorno precoce e efetivo às atividades de vida diária e laborais; terceiro, a redução dos gastos públicos por meio de tratamentos eficientes e eficazes, reduzindo o período de afastamento do paciente em idade produtiva.

Quanto ao número de sessões realizadas, obteve-se uma média de $17,71( \pm 8,6)$ sessões, porém Silva et al. ${ }^{4}$ encontraram uma média menor, de nove $( \pm$ $5,8)$ atendimentos por paciente, contabilizados até a alta fisioterapêutica. Em virtude de somente cinco indivíduos deste estudo terem recebido alta do serviço, pode haver variabilidade deste resultado, ampliando ainda mais a média descrita. 0 referido resultado também é influenciado pela evasão de três usuários durante o tratamento. Considerando as questões econômicas e de saúde pública já descritas anteriormente, a média de sessões realizadas é alta, haja vista que grande parte dos pacientes continua em atendimento. Os fatores de permanência prolongada do paciente no serviço podem englobar uma série de condições, incluindo a diminuição de funcionalidade, a intensidade da dor, a cronicidade dos casos, e aspectos inerentes aos atendimentos prestados, como o número de pessoas envolvidas no tratamento.

Esse número para cada paciente teve média de $5,62( \pm 3,0)$ pessoas. A relação entre paciente e fisioterapeuta é um fator essencial para a adesão do paciente ao tratamento e êxito no processo de reabilitação. No estudo de Subtil et al. ${ }^{14} 11$ pacientes adultos em processo de reabilitação responderam a uma entrevista com três tópicos sobre o processo de adesão na fisioterapia e o papel do relacionamento fisioterapeuta-paciente. Houve relato de críticas em relação à necessidade de maior tempo de contato com os fisioterapeutas e maior atenção aos aspectos emocionais vinculados a desordem física que Ihes acomete. Dessa maneira, verifica-se a importância de construir uma comunicação adequada entre todos os envolvidos no processo de reabilitação, desde o acolhimento do paciente até a alta fisioterapêutica. ${ }^{14}$ No presente estudo, a grande quantidade de profissionais e estudantes da área envolvidos nos atendimentos pode ter dificultado o seguimento de processos objetivos na reabilitação culminando no consequente número elevado de sessões realizadas, quando comparado Silva et al. ${ }^{4}$

O diagnóstico mais prevalente com 38,1\% foi de fratura de tíbia e fíbula, em consonância com os achados de Motoki e Carvalho ${ }^{15}$, em que a perna foi o segmento mais acometido dos membros inferiores, predominando os terços médio (40\%) e distal $(45 \%)$. Já para Domingues e Danaga ${ }^{16}$, as fraturas predominaram os diagnósticos com 71\% dos 186 casos analisados, entretanto as fraturas de maior incidência foram de punho. Os tipos de fratura podem variar conforme o tipo de trauma, sendo considerada uma interrupção completa ou parcial na extensão de um osso. ${ }^{17}$ 
Ao correlacionar o diagnóstico com o sexo, houve predomínio masculino para o diagnóstico de fratura de tíbia e fíbula $(n=6)$, e feminino para artroplastia de joelho $(n=3)$. Manteve, portanto, similaridade com o estudo de Neto et al. ${ }^{18}$, que dos 116 pacientes analisados com diagnóstico de fratura exposta de tíbia grau III, $85 \%$ eram do sexo masculino, e também com os achados de Fernandes et al. ${ }^{19}$, no qual 28 pacientes com diagnóstico de osteoartrite e indicação de artroplastia total de joelho, $75 \%(n=21)$ eram do sexo feminino. A artroplastia total de joelho tem o objetivo de substituir a articulação do joelho por uma prótese metálica. Pacientes com alto nível de dor, alteração na capacidade funcional, processo degenerativo na superfície articular, instabilidade e redução da amplitude articular tem indicação dessa técnica cirúrgica. ${ }^{20}$

Ao avaliar a dor atribuída pelos indivíduos por meio da EVA, observou-se a ocorrência de quadros álgicos variáveis. A dor é responsável por alterar a qualidade de vida e comprometer a capacidade funcional, além de ser grande causadora de absenteísmo e aposentaria por doença. Logo, torna-se razão para uma parcela substancial da população procurar serviços de saúde. Por ser subjetiva, a dor não pode ser avaliada por instrumentos físicos, e para otimizar as intervenções, escalas de avaliação têm sido utilizadas, destacando-se a EVA. Ao considerar a subjetividade do paciente, a EVA permite a validação da dor pelo próprio paciente, descrevendo-a e avaliando-a com exatidão. ${ }^{8,11}$

Grande parte dos distúrbios musculoesqueléticos ocasionam limitações para a execução das atividades. Nos achados de Santos Wendt et al. ${ }^{2}$, incluindo 160 pacientes adultos com alguma desordem musculoesquelética, a amostra indicou que os membros inferiores foram a principal queixa de incapacidade entre os pacientes analisados. A incapacidade representa comprometimento e limitação de atividades. ${ }^{2,21}$

Na presente análise de resultados da escala de LEFS, percebe-se que houve mais respostas positivas que negativas quanto à manutenção da função, o que pode ser relacionado ao fato de que esses pacientes se encontram em situação de atendimento ambulatorial, em fases subagudas e crônicas. No que se refere à correlação entre as escalas
EVA e LEFS, os resultados mostram que não é possivel afirmar que o nível de funcionalidade melhora quando a intensidade da dor diminui, e vice-versa. O mesmo se aplica ao correlacionar a EVA e LEFS com os diagnósticos clínicos mais comuns, fratura de tíbia e fíbula e artroplastia de joelho, não possibilitando afirmar que houve respostas melhores ou piores quanto à funcionalidade ou à dor. Posto isso, verifica-se a importância do fisioterapeuta na reabilitação ortopédica, que contribui para independência dos pacientes nas atividades de vida diária, por meio da melhora da funcionalidade e qualidade de vida. ${ }^{21}$

As limitações para este estudo incluem: número amostral restrito, uma vez que o banco de dados é do ano vigente; desistência, com notificação da evasão de três pacientes durante o tratamento; perda de material, sendo que ao coletar os dados constatou-se que dois prontuários cadastrados não foram encontrados no serviço; e ausência de dados, com a inexistência de aplicação da escala LEFS de um prontuário, não realizada por ocasião da alta fisioterapêutica do paciente.

\section{Considerações finais}

$\infty \times \infty \times \infty \times \infty \times \infty \times \infty \times \infty \times \infty \times \infty \times \infty \times \infty \times \infty$

Por meio da análise dos prontuários dos pacientes em processo de reabilitação no setor de traumatologia, pelo serviço de fisioterapia no CECLIN-UCS/REAB, notou-se que houve prevalência do sexo masculino, e que a idade média dos pacientes é de $40,14( \pm 16,9)$ anos. Entre os cinco pacientes que receberam alta, houve evasão de três durante o tratamento, e aqueles que estão em processo de reabilitação; a média de sessões no total foi de $17,71( \pm 8,6)$; o número de pessoas envolvidas no tratamento fisioterapêutico totalizou com média de $5,62( \pm 3,0)$.

O diagnóstico mais prevalente foi de fratura de tíbia e fíbula, havendo prevalência no sexo masculino. Já a artroplastia de joelho foi mais comum entre as mulheres. Na escala LEFS, a média determinou que é necessário adquirir maior funcionalidade para se tornar independente em diversas atividades de vida diária. Os resultados das correlações para as escalas EVA e LEFS e os diagnósticos 
mais prevalentes, não demostraram ser estatisticamente significativos.

Os resultados obtidos permitem concluir que a continuidade do estudo de avaliação dos prontuários, a partir dos dados já existentes e maior número amostral, bem como as respostas à dor e funcionalidade na condição da alta dos pacientes podem gerar indicadores para o planejamento e acompanhamento de medidas que qualifiquem a intervenção fisioterapêutica e rotinas adotadas na reabilitação.

\section{Referências}

$\infty \times \infty \times \infty \times \infty \times \infty \times \infty \times \infty \times \infty \times \infty \times \infty \times \infty \times \infty \times \infty$

1. Oliveira AC, Braga DLC. Perfil epidemiológico dos pacientes atendidos na clínica de ortopedia da Universidade Paulista. J Health Sci Inst. 2010;28(4):356-8.

2. Wendt AdosS, Chaves AdeO, Urtado CB, Macedo Arde, Reis FJJ, Nogueira LA. Funcionalidade e incapacidade em pacientes comprometimento musculoesquelético. Rev Bras Ciên e Mov. 2017;25(4):15-22.

3. Cerdeira DdeQ, Nunes TTV, Bessa FR, Cavalcanti JM, Magalhães RRG, Souza PJSdeC. Fisioterapia no sertão central do ceará: a caracterização dos pacientes atendidos em um ambulatório de reabilitação funcional. REC. 2013;2(1):24-40.

4. Silva KdeOCda, Oliveira CDRde, Silva MPda, Medeiros YCde, Rodrigues LPC, Leite ECF. Perfil dos pacientes atendidos na clínica escola de fisioterapia no setor de ortopedia e traumatologia, Rev Eletr Estácio Saúde. 2015;4(1):53-8.

5. Assis NL, Bittencourt WS. Perfil Epidemiológico dos Pacientes do Ambulatório de Pneumologia do Hospital Universitário Júlio Muller. Rev Connecti Online. 2012;2(7):56-67.

6. Loureiro BdeCM, Inumaru SMSM, Barreto RR. Perfil epidemiológico e funcional de pacientes com lombalgia crônica. Rev Movimenta. 2017;1(10):43-55.
7. Moreira TS, Sabino GS, De Resende MA. Instrumentos clínicos de avaliação funcional do tornozelo: revisão sistemática. Fisioter Pesq. 2010;17(1):88-93.

8. Bottega FH, Fontana RT. A dor como quinto sinal vital: utilização da escala de avaliação por enfermeiros de um hospital geral. Texto contexto Enfermagem. 2010;19(2):283-90.

9. Pereira LM, Dias JM, Mazuquin BF, Castanhas LG, Menacho MO, Cardoso JR. Tradução, adaptação transcultural e avaliação das propriedades psicométricas do Lower Extremity Functional Scale (LEFS): LEFS-Brasil. [dissertação]. Londrina: Universidade Estadual de Londrina, 2011.

10. Santos JPM, Silva RAda, Fernandes MTP, Frederico RCP, Santos DC, Andraus RAC et al. Uso do questionário Lower Extremity Functional Scale (LEFS-Brasil) em comparação com o Índice Algofuncional de Lequesne para definição de gravidade na osteoartrite de joelho e quadril. Rev Bras Reumatologia. 2017;57(3):274-7.

11. Sacon AB, Pilatt AP, Berbam Lw, Fengler VZ, Bigolin SE. Perfil de sujeitos atendidos na clínica-escola de fisioterapia na área de ortopedia e traumatologia. Rev Contexto Saúde. 2013;11(20):1191-6.

12. Ghisleni MM, Silva VdeCCda, Santos MVdos. Perfil epidemiológico dos pacientes atendidos na área de Ortopedia e Traumatologia da ClínicaEscola de Fisioterapia Univates. Rev Destaques Acadêmicos. 2014;6(3):117-25.

13. Silva LAPda, Ferreira AC, Paulino RES, Guedes GdeO, Cunha MEBda, Peixoto VTCP et al. Análise retrospectiva da prevalência e do perfil epidemiológico dos pacientes vítimas de trauma em um hospital secundário. Rev Med. 2017;4(96):246-54.

14. Subtil MML, Goes DC, Gomes TC, Souza MLde. 0 relacionamento interpessoal e a adesão na fisioterapia. Fisioter Mov 2017;24(4):745-53.

15. Motoki THC, Carvalho KC, Vendramin FS. Perfil de pacientes vítimas de trauma em membro inferior atendidos pela equipe de cirurgia reparadora do Hospital Metropolitano de 
Urgência e Emergência. Rev Bras Cir Plástica. 2013;28(2):276-81.

16. Domingues SV, Danaga AR. Perfil de atendimento fisioterapêutico no ambulatório de ortopedia e traumatologia da santa casa de avarésp. REEC. 2014; 4,(1):1-6.

17. Zago VPA, Grasel CE, Padilha JA. Incidência de atendimentos fisioterapêuticos em vítimas de fraturas em um hospital universitário. Fisioter Mov 2009;22(4):565-73.

18. Neto FCJ, Canal MdeP, Alves BAF, Ferreira PM, Ayres JC, Alves R. Análise das características dos pacientes com fratura exposta de tíbia grau III de Gustilo e Anderson. Rev Bras Ortop. 2016;51(2):143-9.

19. Fernandes DA, Poeta LS, Martins CAdeQ, Lima Fde, Neto FR. Equilíbrio e qualidade de vida após artroplastia total de joelho. Rev Bras Ortop. 2018;53(6):747-53.

20. Ioshitake FACB, Mendes DE, Rossi MF, Rodrigues CDA. Reabilitação de pacientes submetidos à artroplastia total de joelho: revisão de literatura. Rev Fac Ciên Méd Sorocaba. 2016;18(1):11-4.

21. Dantas DRS, Silva Mda, Couto GS, Costa GS, Machado FLdosS, Junior FFUS. Caracterização clínica dos pacientes com distúrbios musculoesqueléticos atendidos em um serviço público de reabilitação fisioterapêutica no município de São Francisco do Conde-Bahia. Rev Ciênc Méd e Biol. 2015;13(2):156-62. 


\section{Anexos}

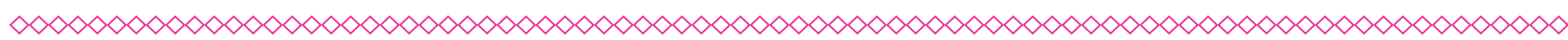

Tabela 1 - Sexo dos indivíduos

\begin{tabular}{|c|c|c|}
\hline Sexo & Quantidade & $\%$ \\
\hline Feminino & 9 & 42,90 \\
\hline Masculino & 12 & 57,10 \\
\hline Total & 21 & 100,00 \\
\hline
\end{tabular}

Fonte: Elaborada pelas autoras 
Tabela 2 - Idade, número de sessões (realizadas e semanal), número de pessoas envolvidas, EVA/LEFS dos indivíduos

\begin{tabular}{lccc} 
& Mínimo & Máximo & Média \\
\hline Idade & 13 & 71 & $40,14( \pm 16,9)$ \\
Número de Sessões Realizadas & 3 & 37 & $17,71( \pm 8,6)$ \\
Número de Pessoas Envolvidas & 2 & 14 & $5,62( \pm 3,0)$ \\
Número Semanal de Sessões & 2 & 4 & $2,24( \pm 0,5)$ \\
EVA & 0 & 10 & $4,67( \pm 3,0)$ \\
LEFS & 14 & 58 & $58,90( \pm 13,8)$ \\
\hline
\end{tabular}

Fonte: Elaborada pelas autoras 
Tabela 3 - Diagnóstico dos indivíduos e divisão por sexo

\begin{tabular}{lcccc} 
Diagnóstico & Frequência & $\%$ & \multicolumn{2}{c}{ Sexo } \\
Fratura tíbia/fíbula & 8 & 38,1 & 2 & 6 \\
Artroplastia de joelho & 5 & 23,8 & 3 & 2 \\
Fratura de patela & 1 & 4,8 & 0 & 1 \\
Fratura do pé & 1 & 4,8 & 0 & 1 \\
Fratura plato tibial & 1 & 4,8 & 1 & 0 \\
Artroplastia de quadril & 1 & 4,8 & 1 & 0 \\
Instabilidade femoropatelar & 1 & 4,8 & 0 & 1 \\
Pós-operatório de ligamento cruzado & 1 & 4,8 & 1 & 0 \\
anterior + menisco & & & & \\
Pós-operatório de reconstrução do & 1 & 4,8 & 1 & 0 \\
ligamento femoropatelar & 1 & 4,8 & 0 & 1 \\
Pós-operatório de tenoplastia patelar & & & \\
\hline
\end{tabular}

Fonte: Elaborada pelas autoras 
Figura 1 - Correlação entre LEFS e EVA

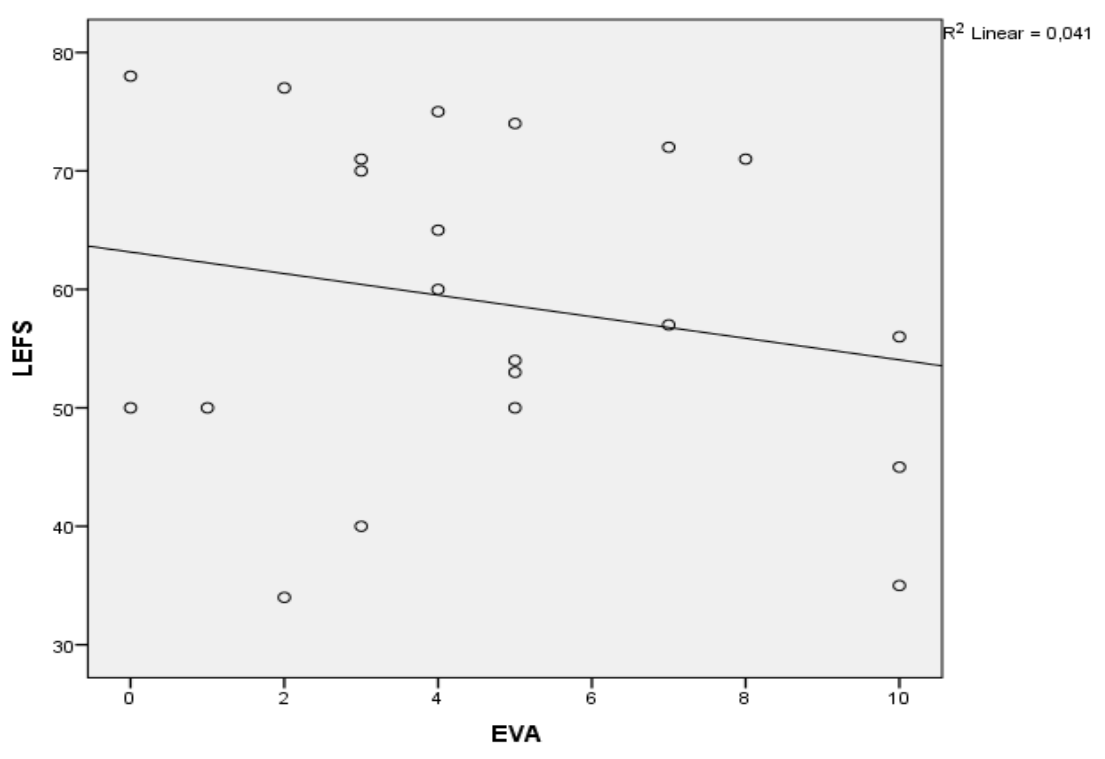

Fonte: Elaborada pelas autoras

(clique para voltar ao texto) 